(C) 2016 IEEE. Personal use of this material is permitted. Permission from IEEE must be obtained for all other uses, in any current or future media, including reprinting/republishing this material for advertising or promotional purposes, creating new collective works, for resale or redistribution to servers or lists, or reuse of any copyrighted component of this work in other works. 


\title{
On the Quality of the Gaussian Copula for Multi- temporal Decision-making Problems
}

\author{
Ricardo J. Bessa \\ Center for Power and Energy Systems (CPES) \\ INESC TEC - INESC Technology and Science \\ Porto, Portugal \\ ricardo.j.bessa@inesctec.pt
}

\begin{abstract}
Multi-temporal decision-making problems require information about the potential temporal trajectories of wind generation for a given time horizon. Typically, the Gaussian copula is used for modelling the dependency between probabilistic forecasts from different lead-times. This paper explores the vine copula framework as a benchmark model since it captures complex multivariate dependence structures with mixed types of dependencies. The results show that a Gaussian copula with a suitable covariance matrix suffice to generate high quality temporal trajectories.
\end{abstract}

Index Terms--Wind power, uncertainty, decision-making, copula, vines, temporal trajectories.

\section{INTRODUCTION}

The large-scale integration of wind generation into power systems operation requires innovative tools for handling its uncertainty and variability, which, in general, consists in decision-making problems under uncertainty. Problems, such as the unit commitment [1] and wind power-storage coordination [2], are inserted in multi-temporal stochastic optimization paradigm.

The wind power forecast is a key input of these tools, which, during the last twenty years, has advanced from point forecast to different approaches for modeling and communicating forecast uncertainty; for a literature review see [3]. Traditionally, multi-temporal stochastic optimization problems use a set of scenarios to characterize the uncertainty associated to load and generation. Therefore, one possible approach to integrate wind power uncertainty into these problems is through a set of temporal trajectories of wind generation for the next hours or days. For example, Haessig et al. [2] shows the importance of including the temporal dependency of errors in multi-temporal problems; discarding autocorrelation of forecast errors can lead to underestimation of storage capacity.

The first contribution on this topic was the short-term scenarios proposed by Pinson et al. [4]. The method is inspired by the Normal-to-Anything (NORTA) method [5] and generates a set of random vectors (short-term scenarios or temporal trajectories) from a Gaussian copula. The covariance matrix of the copula is updated as new observations are made available in a time-adaptive framework. Morales et al. [6] also used the Gaussian copula to characterize the time and spatial dependencies of wind speed at different geographical locations.

Ma et al. [7] proposed a method to generate scenarios containing temporal information about wind power variability (but only first order differences) and uncertainty. An exponential covariance function, like in [8], is used in the Gaussian copula. Finally, Tastu et al. [9] extend this framework to generate joint predictive densities of wind power output (and ultimately, spatial-temporal trajectories). The proposed method also relies on the Gaussian copula for the dependency structure and takes advantage of the sparsity of precision matrices (i.e., inverse covariance matrix), accounts for non-constant conditional variances and directiondependent conditional dependencies. The Gaussian copula was also used in transmission network planning [10] and for modelling the wind power Europe-wide and its effects on the Swiss power grid [11].

Although not related to modelling wind power forecast uncertainty, Díaz [12] shows that Gumbel copulas, when compared to Gaussian copulas, seem to be only appropriated for representing wind power in a bivariate form (e.g., dependency between two wind farms). Conversely, Louie [13] studied which bivariate copula families were more suited to model wind power dependency structures and found that Gaussian dependence may not be justified and the use of Gumbel copulas can improve the models.

The research question is whether the Gaussian copula is appropriated or not to represent multi-temporal dependencies of uncertainty forecasts. The vine copula framework is based on factorizing a d-dimensional density into a product of $d(d$ 1)/2 pair-copulas [14]. This framework is appropriate for modelling complex multivariate dependence structures with mixed types of dependencies, such as asymmetries and tail dependencies, since each pair-copula can belong to a different parametric copula function. Therefore, it is very flexible and a

This work is financed by the ERDF - European Regional Development Fund through the Operational Programme for Competitiveness and Internationalisation - COMPETE 2020 Programme within project «POCI01-0145-FEDER-006961», and by National Funds through the FCT Fundação para a Ciência e a Tecnologia (Portuguese Foundation for Science and Technology) as part of project UID/EEA/50014/2013. 
perfect benchmark model to study if the Gaussian copula provides a suitable approximation or not for the dependency structure. Vine copulas are being widely used in the financial and insurance sectors [15], with very few applications to the energy sector. For instance, in [16], the vine copulas are used for spatial-temporal modelling of auto and cross-correlation between different wind power time series.

The present paper aims to explore the flexibility provided by the vine copula to generate temporal trajectories of wind power and compare their quality with the ones generated from a Gaussian copula. The following two original contributions are produced: (a) first application of the vine copula approach to generate temporal trajectories of wind power forecast uncertainty; (b) comparison between vine copula and Gaussian copula for three real wind farms, using the evaluation framework from [8] and the scoring rule from [17].

The remaining of this paper is organized as follows: section II presents a brief introduction to the vine copula framework; section III describes how temporal trajectories of wind power can be generated; section IV describes the Gaussian copula method; section V presents the numerical and evaluation results for three real wind farms; conclusions are given in section VI.

\section{THE VINE COPULA FRAMEWORK}

\section{A. Copula Definition}

A copula provides a way of separating the marginal distributions from the multivariate dependency structure and links these two together to form the joint distribution [14]. The Sklar's theorem argues that every d-dimensional distribution function $F$ with marginals $F_{i}\left(x_{i}\right), \mathrm{i}=1 \ldots \mathrm{d}$, can be written as [18]:

$$
F\left(x_{1}, \ldots, x_{d}\right)=C_{1 \ldots d}\left[F_{1}\left(x_{1}\right), \ldots, F_{d}\left(x_{d}\right)\right]
$$

where $x_{i}$ is a vector of random variables (r.v.) and $C$ is a copula. If each $F_{i}\left(x_{i}\right)$ is continuous then $C$ is unique. In words, Eq. 1 means that it is possible to describe the joint distribution of $x_{1}, \ldots, x_{d}$ by the marginal distributions $F_{i}\left(x_{i}\right)$ and the copula $C$ that represents the dependency structure between the marginal.

The right-hand-side shows that a copula is the joint distribution of r.v. $u_{1}, \ldots, u_{p}$ [note that $\left.u_{i}=F_{i}\left(x_{i}\right)\right]$, which are marginally uniformly distributed as $U(0,1)$. Therefore, it is also possible to write Eq. 1 as follows:

$$
C_{1 \ldots d}\left(u_{1}, \ldots, u_{d}\right)=F\left[F_{1}^{-1}\left(u_{1}\right), \ldots, F_{d}^{-1}\left(u_{d}\right)\right]
$$

where $F_{i}^{-1}$ is the inverse of the marginal distributions.

By applying the chain rule on Eq. 1, the joint density function is given by:

$$
\begin{aligned}
& f\left(x_{1}, \ldots, x_{d}\right)=\frac{\partial^{2}}{\partial x_{1} \cdots \partial x_{d}} C_{1 \cdots d}\left(u_{1}, \ldots, u_{d}\right) \\
& =f_{1}\left(x_{1}\right) \cdots \cdots f_{d}\left(x_{d}\right) \cdot c_{1 \cdots d}\left(u_{1}, \ldots, u_{d}\right)
\end{aligned}
$$

where $c_{1 \ldots d}$ is a d-dimensional copula density.
There are a vast number of copula parametric functions in the literature, such as the Archimedean (e.g., Clayton, Gumbel, Frank) and elliptical (Gaussian, Student-t) [14].

\section{B. Joint Density Decomposition}

Any joint density function can be factorized into a product of density functions [19][20], as follows:

$$
\begin{aligned}
& f\left(x_{1}, \ldots, x_{d}\right)= \\
& f_{d}\left(x_{d}\right) \cdot f\left(x_{d-1} \mid x_{d}\right) \cdot f\left(x_{d-2} \mid x_{d-1}, x_{d}\right) \cdots \cdots f\left(x_{1} \mid x_{1}, \ldots, x_{d}\right)
\end{aligned}
$$

where $f(x \mid$.) is the conditional density function. For three variables, it becomes:

$$
f\left(x_{1}, x_{2}, x_{3}\right)=f_{3}\left(x_{3}\right) \cdot f\left(x_{2} \mid x_{3}\right) \cdot f\left(x_{1} \mid x_{2}, x_{3}\right)
$$

Note that this decomposition is different in case of a reordering of the variables indices $(1 \ldots d)$, thus is not unique.

The right-hand-side of Eq. 4 shows that a joint density function decomposed into a product of marginal densities [i.e., $\left.f_{d}\left(x_{d}\right)\right]$ and terms describing the dependency between the r.v. [e.g., $\left.f_{d}\left(x_{d-1} \mid x_{d}\right)\right]$.

Note that a conditional density function, considering the copula definition of Eq. 3, is given by:

$$
f\left(x_{d-1} \mid x_{d}\right)=\frac{f_{d-1, d}\left(x_{d-1}, x_{d}\right)}{f_{x_{d}}\left(x_{d}\right)}=f_{d-1}\left(x_{d-1}\right) \cdot c\left(F_{d-1}\left(x_{d-1}\right), F_{d}\left(x_{d}\right)\right)
$$

In [20] it is shown that any conditional term in Eq. 4 can be decomposed into a form similar to Eq. 6 using the following general formula:

$$
f(x \mid \mathbf{v})=c_{x v_{j} \mid v_{-j}}\left(F\left(x \mid \mathbf{v}_{-j}\right), F\left(v_{j} \mid \mathbf{v}_{-j}\right)\right) \cdot f\left(x \mid \mathbf{v}_{-j}\right)
$$

where $\boldsymbol{v}$ is a $d$-dimensional vector, $j$ is one arbitrarily chosen component of $\boldsymbol{v}$ and $\boldsymbol{v}_{-j}$ is the $\boldsymbol{v}$ vector excluding the $j$-th component. For the three variables example, this equation becomes:

$$
\begin{aligned}
& f\left(x_{1} \mid x_{2}, x_{3}\right)=c_{1,3 \mid 2}\left(F\left(x_{1} \mid x_{2}\right), F\left(x_{3} \mid x_{2}\right)\right) \cdot f\left(x_{1} \mid x_{2}\right) \\
& =c_{1,3 \mid 2}\left(F\left(x_{1} \mid x_{2}\right), F\left(x_{3} \mid x_{2}\right)\right) \cdot f\left(x_{1}\right) \cdot c_{1,2}\left(F\left(x_{1}\right), F\left(x_{2}\right)\right)
\end{aligned}
$$

Details about the calculation of $F(x \mid v)$ are given in [20].

Replacing Eq. 8 in Eq. 5 and using Eq. 6 to decompose $f\left(x_{2} \mid x_{3}\right)$, the following decomposition is obtained for an example with three r.v.:

$$
\begin{gathered}
f\left(x_{1}, x_{2}, x_{3}\right)=c_{1,3 \mid 2}\left(F\left(x_{1} \mid x_{2}\right), F\left(x_{3} \mid x_{2}\right)\right) \cdot c_{1,2}\left(F_{1}\left(x_{1}\right), F_{2}\left(x_{2}\right)\right) . \\
\cdot c\left(F_{2}\left(x_{2}\right), F_{3}\left(x_{3}\right)\right) \cdot f\left(x_{1}\right) \cdot f\left(x_{2}\right) \cdot f_{3}\left(x_{3}\right)
\end{gathered}
$$

From Eq. 9 it is possible to see that the joint density can be expressed as a product of pair-copulas acting on several different conditional probability distributions and marginal density functions. Note that each pair-copula can be chosen independently from the others, which provides high flexibility in dependency modelling. 


\section{Vine Structure}

The vine copula framework, defined as a graphical tool for dependent r.v., was firstly proposed by Bedford and Cooke [19]. The seminal work of Aas et al. [20] established the statistical background for likelihood-based inference, model selection and simulation of random vectors, which enabled its practical application.

A vine is a graphical representation that specifies the paircopula construction of Eq. 4 and consists in acyclic connected graphs with nodes and edges. For an example with four r.v., Fig. 1 illustrates two types of vines, drawable vine (D-vine) and canonical vine (C-vine). In a D-vine, each node is connected to a maximum of two nodes. Conversely, in a Cvine there is a unique node connected to $d-j$ edges. Each vine type consists of $d-1$ trees with tree $T_{j}$ having $(d+1)-j$ nodes and $d-j$ edges, e.g. four r.v. $(d=4)$ results in three trees $(j=1,2,3)$ and tree $T_{2}$ has 3 nodes and 2 edges. The nodes in tree $T_{j}$ are used to define the labels of the edges in tree $T_{j+l}$. The edges in $T_{j}$, which become nodes in $T_{j+1}$, are joined by an edge in $T_{j+1}$ only if these edges in $T_{j}$ share a common node.

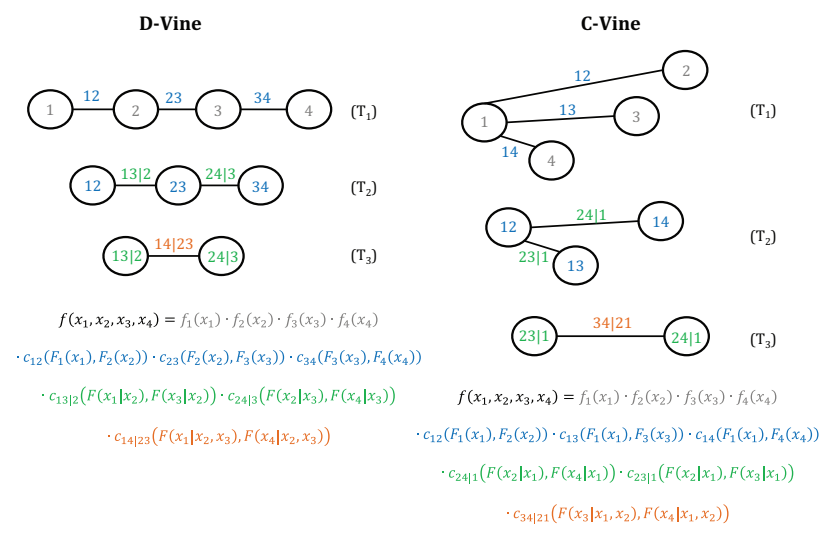

Figure 1. Drawable vine (D-vine) and canonical vine (C-vine) copulas for an example with four r.v.

As can be seen in Fig. 1, this graphical representation is a useful tool to represent the joint density decomposition $f\left(x_{1}, x_{2}, x_{3}, x_{4}\right)$ of Eq. 4. The nodes of the first tree $\left(T_{1}\right)$ correspond to marginal density functions in Eq. 9 and each edge corresponds to a pair-copula density, e.g. the edge between nodes 12 and 23 in tree $T_{2}$ results in copula density $c_{13 \mid 2}$. Finally, note that the sequence of the nodes defines a different manner of decomposing the joint density. [20]:

The joint density $f\left(x_{1}, \ldots, x_{d}\right)$ for a D-vine copula is given by

$\prod_{k=1}^{d} f\left(x_{k}\right) \prod_{j=1}^{d-1} \prod_{i=1}^{d-j} c_{i, i+j \mid i+1, \ldots, i+j-1}\left\{F\left(x_{i} \mid x_{i+1}, \ldots, x_{i+j-1}\right), F\left(x_{i+j} \mid x_{i+1}, \ldots, x_{i+j-1}\right)\right\}$

and for a $\mathrm{C}$-vine is

$$
\prod_{k=1}^{d} f\left(x_{k}\right) \prod_{j=1}^{d-1} \prod_{i=1}^{d-j} c_{j, j+i \mid 1, \ldots, j-1}\left\{F\left(x_{j} \mid x_{1}, \ldots, x_{j-1}\right), F\left(x_{j+i} \mid x_{1}, \ldots, x_{j-1}\right)\right\}
$$

After defining the vine type and r.v.'s order, which should be based on expert knowledge and trial-error experiences, the pair-copula type and corresponding parameters are estimated from data with the method described in section III and with the help of R package "CDVine" [21].

\section{GeNeration OF Wind POWER TEMPORAL TRAJECTORIES}

This section describes the necessary steps to generate wind power temporal trajectories using the vine copula framework. The r.v. are marginal predictive functions obtained from any probabilistic forecast method, such as [22], and the number of r.v. corresponds to the forecast time horizon (T).

The preliminary steps consist in model section, i.e. choosing between $\mathrm{C}$-vine and D-vine and defining the order of the r.v. Note that for a D-vine there are $d$ ! possible ways of ordering the r.v. The model selection is based on expert knowledge and the fact that this problem consists in time trajectories and the strongest dependencies in the first tree are between lead-times (or r.v.) close in time (see for instance [4]). Therefore, a natural choice is a D-vine with the r.v. in the tree $T_{1}$ nodes ordered by its temporal sequence, i.e. $t+1$, $t+2, \ldots, t+T$. Note that a $\mathrm{C}$-vine is more suitable for dependency structures where one r.v. has a strong influence in the others.

\section{A. Pair-copula Selection}

In order to apply any copula based method, the data must lie in the unit hypercube. This basically consists in the following transformation:

$$
u_{t+k}=\hat{F}_{t+k \mid t}\left(y_{t+k}\right)
$$

where $u_{t+k}$ is the uniform variable, $\hat{F}_{t+k i t}$ the marginal predictive function for lead-time $t+k$ and generated at time instant $t$ and $y_{t+k}$ the observed wind power.

The pair-copula type to use in each edge can be determined by plotting the data from Eq. 12 combined with a goodness-of-fit test.

Fig. 2 shows two bivariate plots between the uniform variables of different lead-times $(t+1$ and $t+2 ; t+27$ and $t+28)$ that represent two edges from the first tree. The data used for these plots is from the wind farm dataset described in section $\mathrm{V}$. The plot in the middle is a set of random numbers generated from a Gaussian bivariate copula with the correlation parameter calculated from the $t+1$ and $t+2$ data and it is used for comparison. This plot shows that, visually, both bivariate dependence structures are different from the Gaussian, mainly characterized by upper and lower tail dependency (i.e., high concentration of points in the tails). Note that the Gaussian copula does not have tail dependency.

This visual inspection provides an indication about the copula type. However, a goodness-of-fit test should be applied to select one copula function from a vast set. Several tests and visualization tools are discussed in [21] to analyse bivariate data. For instance, using the Clarke test, the selected copula for the dependency between $u_{t+1}$ and $u_{t+2}$ was the Frank 
copula, while for $u_{t+27}$ and $u_{t+28}$ was the Student-t copula. In both cases, the Gaussian copula received a low score in test statistic.
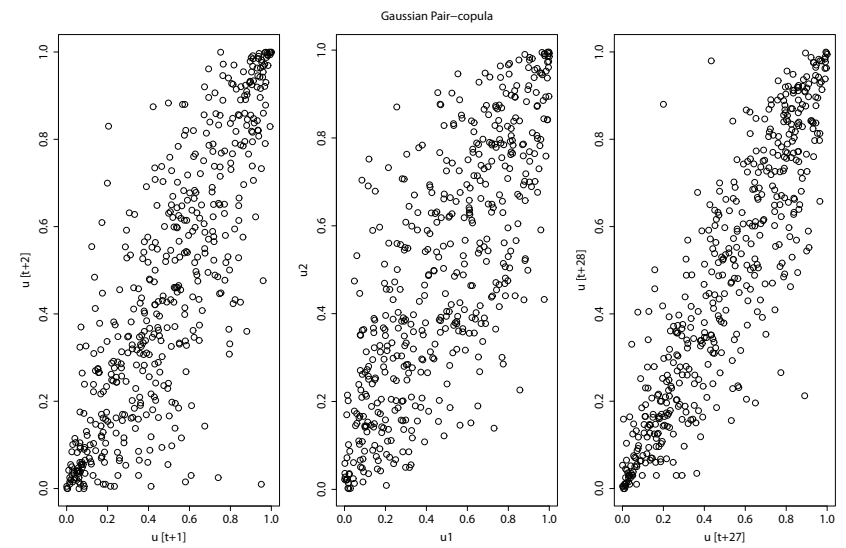

Figure 2. Bivariate plots between uniform variables of different lead-times.

In [20] and [21] an iterative process is proposed to select the pair-copula of each edge and estimate the corresponding parameter(s). The procedure basically consists of fitting all available copulas using maximum likelihood estimation, then the Akaike or Bayesian Information Criteria (AIC and BIC, respectively) are calculated for each fitted copula, and finally the copula with minimum AIC or BIC is selected for that specific edge.

It is this flexibility in combining different types of copulas that makes this approach theoretically advantageous over the classical Gaussian copula.

\section{B. Model Inference}

After selecting the copula type for each pair-copula and with the available dataset, the next step consists of estimating the parameters of all pair-copulas. The following sequential estimation procedure is described in [20]:

1. determine the copula type to use in each edge from tree $T_{l}$ by using the method described in section III.A;

2. estimate the parameters of the selected copula by using the transformed original data (Eq. 12);

3. using the copula's parameters estimated in step (2) for tree $T_{1}$ and the function $F(x \mid v)$ (see Eq. 8 and reference [20]), compute the observations required to estimate the copula parameters in tree $T_{2}$;

4. determine the copula type to use in each edge from tree $T_{2}$;

5. iterate.

This sequential procedure is implemented in the function "CDVineCopSelect" from the R Package "CDVine" [21]. However, this procedure does not guarantee a global optimal fit. In order to tackle this problem, the Evolutionary Particle Swarm Optimization (EPSO) [23] was used in this paper to maximize the global log-likelihood of the vine. EPSO, as a population-based method, relies on a set of moving solutions denoted as particles. In this case, a particle is a set of parameters associated to each pair-copula. The fitness function is the log-likelihood function of a D-vine [20]:

$$
\sum_{j=1}^{d-1} \sum_{i=1}^{d-j} \sum_{z=1}^{T} \log \left[c_{i, i+j i l+1, \ldots, i+j-1}\left(F\left(u_{i, z} \mid u_{i+1, z}, \ldots, u_{i+j-1, z}\right), F\left(u_{i+j, z} \mid u_{i+1, z}, \ldots, u_{i+j-1, z}\right)\right)\right]
$$

Note that for each copula in Eq. 13, at least one parameter needs to be determined, so the size of the particles is related to the size of the D-vine.

Typically, the EPSO algorithm initiates the particles by randomly generating a population of solutions as a set of random number from $m$-dimensional space ( $m$ is the number copula parameters). In this case, in order to reduce the search space of the algorithm and increasing its efficiency, the solution from the sequential estimation is associated to one particle, while the other particles have the sequential solution perturbed by a Gaussian distribution with mean zero and standard deviation equal to 0.2 .

The limits of the copula's parameters, listed in [21], are enforced by the EPSO algorithm. A parallel evaluation of the fitness function was implemented using the $\mathrm{R}$ Package "doParallel" [24]. The average running time with EPSO is 79 minutes (in contrast to 6120 minutes with the Limitedmemory BFGS method).

\section{Generation of Temporal Trajectories}

After determining the pair-copula and parameters of the Dvine structure, the generation of random vectors is straightforward. First, $d$-samples, $w_{l} \ldots w_{d}$, are generated from a uniform distribution; then, the following relations are established:

$$
\begin{array}{ccc}
u_{1}= & w_{1} \\
u_{2}= & F^{-1}\left(w_{2} \mid u_{1}\right) \\
u_{3}= & F^{-1}\left(w_{3} \mid u_{1}, u_{2}\right) \\
\cdots & = & \ldots \\
u_{d}= & F^{-1}\left(w_{d} \mid u_{1}, \ldots, u_{d-1}\right)
\end{array}
$$

where $F^{-1}\left(w_{d} \mid u_{1}, \ldots, u_{d-1}\right)$ is given by the function $F(x \mid v)$ (see Eq. 8). The detailed description of the simulation loop for a D-vine can be found in [20] and for this paper the function "CDVineSim" from the R Package "CDVine" was used to generate $N$ temporal trajectories (or random vectors). Note that these trajectories are in the unit hypercube, thus the following relation is used to convert the values into wind power values:

$$
y_{t+k}^{[n]}=\hat{F}_{t+k i t}^{-1}\left(u_{t+k}\right)
$$

Fig. 3 illustrates a set of 100 temporal trajectories sampled from a D-vine fitted with the procedure described in this section from one wind farm of dataset described in section $\mathrm{V}$.

\section{GAUSSIAN COPUla MethoD}

The Gaussian copula method generates $N$ temporal trajectories of wind power using the following process (described in [4] and [5]): 
1. generates $N$ random vectors $Z$ from a multivariate Gaussian distribution (i.e., Gaussian copula) with zero mean and covariance matrix $\Sigma_{Z}$;

2. transforms $Z$ with Eq. 16 to obtain a random vector;

$$
y_{t+k}^{[n]}=\hat{F}_{t+k \mid t}^{-1}\left(\Phi\left(Z_{t+k}^{[n]}\right)\right)
$$

where $\Phi$ is the distribution function of a standard normal r.v.

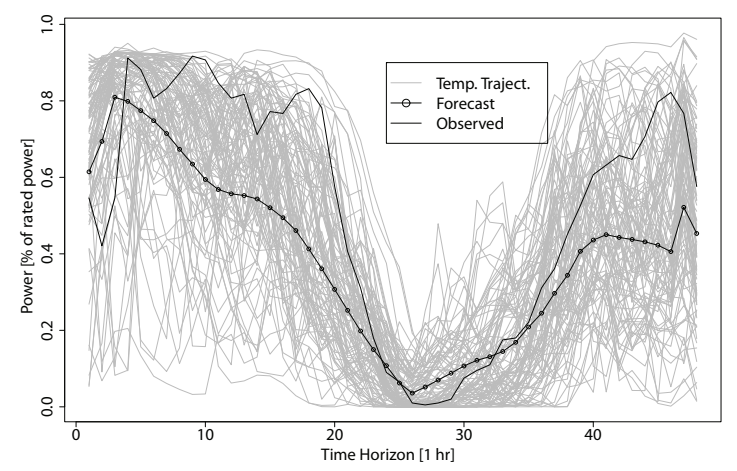

Figure 3. Set of 100 wind power temporal trajectories sampled from a Dvine.

The dependency structure between the lead-times is modelled with a Gaussian copula and, in this paper, two types of covariance matrices were considered:

a) The Pearson's correlation coefficient is used to compute the empirical covariance of variable $Z_{t}$ defined as follows:

$$
Z_{t}=\Phi^{-1}\left(\hat{F}\left(P_{t}\right)\right)
$$

The covariance matrix is computed from a learning sample of past observations of $Z$.

b) Exponential covariance function is given by

$$
\operatorname{cov}\left(Z_{t+k_{1}}, Z_{t+k_{2}}\right)=\exp \left(-\frac{\left|k_{1}-k_{2}\right|}{v}\right)
$$

where $Z_{t+k l}$ is the Gaussian r.v. for lead time $t+$ $k_{l}$ and where $v$ is the range parameter controlling the strength of the correlation of r.v. among the set of lead times.

\section{NUMERICAL RESULTS}

This section presents numerical results with respect to the quality (measured by different scoring rules) of the temporal trajectories generated with different dependency structures.

\section{A. Case Study Description}

The case-study consists of the first three real wind farms from the Global Energy Forecasting Competition (GEFCOM 2012) dataset, which is freely available in [25]. Three years of data are available and consists of historical power measurements and weather predictions extracted from the European Centre for Medium-range Weather Forecasts model (ECMWF) with hourly time resolution. The wind power values were normalized between 0 and 1 by the respective rated power of the wind farms.

The last year was used to generate a set of wind power trajectories and corresponding evaluation. The time horizon is 48 hours-ahead. The marginal predictive distributions are from the probabilistic method described in [22].

The EPSO parameters used in this paper were: mutation rate $(0.3)$; communication probability $(0.7)$; number of particles (30); stopping criteria (20 iterations with the same global maximum or 1000 iterations).

\section{B. Multivariate Skill Score}

In the literature, it is possible to find several skill scores for evaluating univariate probabilistic forecasts, such as the Continuous Ranking Probability Score (CRPS). However, for multivariate probabilistic forecasts, the number of skill scores remains very limited. The mostly used skill score for meteorological ensemble evaluation is the energy score (ES) proposed in [26]. This score is a generalization of the univariate CRPS for multivariate quantities and it is given by:

$$
E S=\frac{1}{J} \sum_{j=1}^{J}\left\|\mathbf{y}-\hat{\mathbf{y}}^{[j]}\right\|_{2}-\frac{1}{2 \cdot J^{2}} \sum_{i=1}^{J} \sum_{j=1}^{J}\left\|\hat{\mathbf{y}}^{[i]}-\hat{\mathbf{y}}^{[j]}\right\|_{2}
$$

where $\left\|_{\|}\right\|_{2}$ is an $L_{2}$ norm, $y$ is the observed time trajectory of wind power, $\hat{\mathbf{y}}^{[j]}$ is the $j$-th temporal trajectory and $J$ is the number of sampled trajectories. The ES is averaged over the entire evaluation dataset (i.e. 365 days).

In [8] and [27] it is shown that the ES has low discrimination ability when focusing on the dependence structure of multivariate probabilistic forecasts. Recently, it is proposed in [17] a new score, called p-variogram score (VSp). This skill score is based on pairwise differences between all components of the multivariate variable and, compared to the ES, possesses much better discrimination ability with respect to correlations between the different components. The VS-p is calculated as follows:

$$
\mathrm{VS}-\mathrm{p}=\sum_{i, j=1}^{d} w_{i j}\left(\left|y_{i}-y_{j}\right|^{p}-\frac{1}{J} \sum_{z=1}^{J}\left|\hat{y}_{i}^{[z]}-\hat{y}_{j}^{[z]}\right|^{p}\right)
$$

where $y_{i}$ and $y_{j}$ are the $i$-th and $j$-th component of the observed wind power trajectory, $\hat{y}_{i}^{[z]}$ and $\hat{y}_{j}^{[z]}$ are the $i$-th and $j$ th component of the $z$-th trajectory, $w_{i j}$ are positive weights defined by an expert to put more emphasis on a certain component combination and $p$ is the power order. The VS-p is averaged over the entire evaluation set.

In this paper, the weights $w_{i j}$ were set equal to the inverse of time distance between components $i$ and $j$, i.e. $1 /\|\mathrm{i}-\mathrm{j}\|$. Results presented in [17] show that a VS-p with $p \leq 1$ has the best discriminative ability. 
Tables I and II compare the ES and VS-p obtained by the D-vine copula and the following dependency structures: (a) independent copula; (b) Gaussian copula with empirical covariance matrix (similar to [4] but without the time-varying covariance); (c) Gaussian copula with exponential covariance (as described in [7]). These metrics are evaluated on 1000 trajectories. Note that the parameter of the exponential covariance matrix was determined by trial-error experiences: 10, 7 and 5 for each wind farm correspondingly.

The ES results in table I show its low discrimination ability since the difference to the independent copula is minor, only around $2 \%$. Considering the ES metric, the D-vine achieves the lowest values for the two wind farms. The Gaussian copula with exponential covariance presents an ES lower than the empirical covariance.

The discrimination ability of the VS-p metric is higher than the ES, e.g. the D-Vine improves over the independent copula around $33 \%$. An interesting result is that the Gaussian copula with exponential covariance presents a VS-p lower than the one obtained with the D-vine for the two wind farms. This result shows that the quality of the temporal trajectories cannot be evaluated with a single skill score. As mentioned in [26], the ES is often not sufficiently sensitive to misspecifications of the correlations between the different components.

The results in Table II (wind farm 3) are similar, but in this case the D-vine presents the lowest value in both ES and VSp. The temporal trajectories generated with the exponential covariance matrix present a better quality than the ones generated with the empirical covariance matrix.

In summary, the results for the three wind farms show that the Gaussian copula can lead to high quality temporal trajectories, as long as a proper structure for the covariance matrix is selected. The D-vine is also capable of generating trajectories with high quality, based on its flexibility to represent different dependency structures. It is important to stress that both Gaussian copula with empirical covariance matrix and D-Vine need a large dataset of historical data to make an accurate estimation of the correlation statistics, while the exponential covariance does have this requirement. This fact might explain the better performance from the later dependency structure.

TABLE I

ENERGY SCORE (ES) AND P-VARIOGRAM SCORE (VS-P) FOR DIFFERENT DEPENDENCY STRUCTURES AND WIND FARMS 1 AND 2.

\begin{tabular}{ccccccc}
\hline \hline & \multicolumn{3}{c}{ Wind Farm 1 } & \multicolumn{3}{c}{ Wind Farm 2 } \\
\hline $\begin{array}{c}\text { Dependency } \\
\text { Structure }\end{array}$ & ES & VS-0.5 & VS-1 & ES & VS-0.5 & VS-1 \\
\hline $\begin{array}{c}\text { Independent } \\
\text { Gaussian Copula } \\
\text { (Emp. Cov.) }\end{array}$ & 0.7486 & 11.81 & 6.54 & 0.7159 & 11.36 & 7.11 \\
$\begin{array}{c}\text { Gaussian Copula } \\
\text { (Exp. Cov.) }\end{array}$ & 0.7361 & 7.98 & 4.39 & 0.7029 & 7.90 & 5.09 \\
D-Vine Copula & $\underline{\mathbf{0 . 7 3 5 4}}$ & $\mathbf{7 . 8 5}$ & 4.34 & $\underline{\mathbf{0 . 7 0 2 5}}$ & 7.83 & 5.07 \\
\hline \hline
\end{tabular}

C. Event-based Evaluation

This section evaluates the quality of the temporal trajectories using the event-based methodology proposed in [8]. The following gradient event was considered: maximum absolute variation in the process over a window of length $h$, centered on lead-time $k$, being (or not) greater than $t h r$. For this event, the Brier Score (BS) was calculated as a function of the lead-time $k$. Fig. 4 depicts the BS for wind farm 1 considering two combinations of parameters: (a) $h=6$; $t h r=0.2$; (b) $h=6 ; t h r=0.2$. Note better performance corresponds to higher BS score.

TABLE II

\begin{tabular}{|c|c|c|c|}
\hline \multicolumn{4}{|l|}{ DEPENDENCY STRI } \\
\hline \multirow[b]{2}{*}{ Dependency Structure } & \multicolumn{3}{|c|}{ "Wind Farm 3} \\
\hline & ES & VS-0.5 & VS-1 \\
\hline Independent & 0.8179 & 12.72 & 8.40 \\
\hline $\begin{array}{l}\text { Gaussian Copula } \\
\text { (Emp. Cov.) }\end{array}$ & 0.8079 & 9.63 & 6.58 \\
\hline $\begin{array}{l}\text { Gaussian Copula } \\
\text { (Exp. Cov.) }\end{array}$ & 0.8077 & 9.58 & 6.55 \\
\hline D-Vine Copula & $\underline{0.8075}$ & $\underline{9.52}$ & $\underline{6.54}$ \\
\hline
\end{tabular}
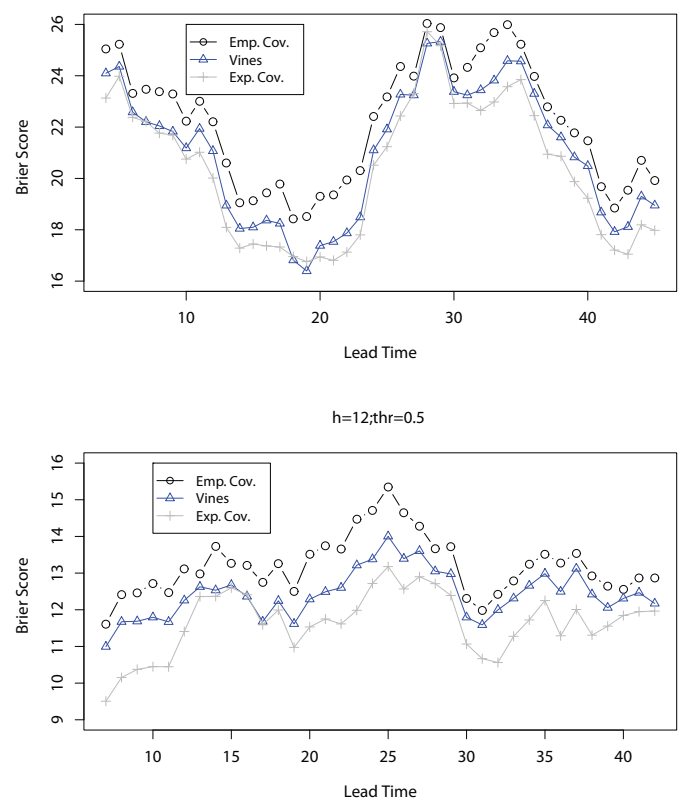

Figure 4. Brier score as a function of lead-time for wind farm 1.

The results in Fig.4 confirm the ones presented in Table I for the VS-p. Also in the BS, the skill of the trajectories generated by the Gaussian copula with exponential covariance is higher than the one obtained with the D-vine. The difference in terms of BS is higher for the second gradient event. Fig. 5 presents the BS results for wind farm 3. In contrast to the results presented in table II, for these two events it is not possible to conclude that the D-vine outperforms the Gaussian with exponential matrix. Nevertheless, for the second gradient event the D-vine presents the lowest BS during some lead-times.

\section{CONCLUSIONS}

This paper presents a first application of the vine copula framework to generate a set of wind power temporal 
trajectories (associated to wind power forecast uncertainty) that can be included in decision-making problems under risk. Using different skill scores, the trajectories generated with the vine copula approach were compared with the Gaussian copula for three real wind farms.

The results show that the Gaussian copula is a reasonable model for the temporal dependency of forecast errors, as long as a suitable structure is selected for the covariance matrix. For the three wind farms analyzed in this paper, the Gaussian copula with exponential covariance matrix outperformed the vine copula for two out of three wind farms. The main advantage of the Gaussian copula is its mathematical simplicity, while the advantage of the vine copula framework is its flexibility. For instance, the analyst does not need to find the "best" dependency structure (i.e., type of covariance matrix). The main advantage of the exponential covariance matrix is that it does not require a large historical dataset, which may justify the higher performance in two wind farms.

Topics for future work are: (a) compare the quality of temporal trajectories generated with different methods in a specific power system management process; (b) explore the vine copula framework for modeling spatial-temporal uncertainty.
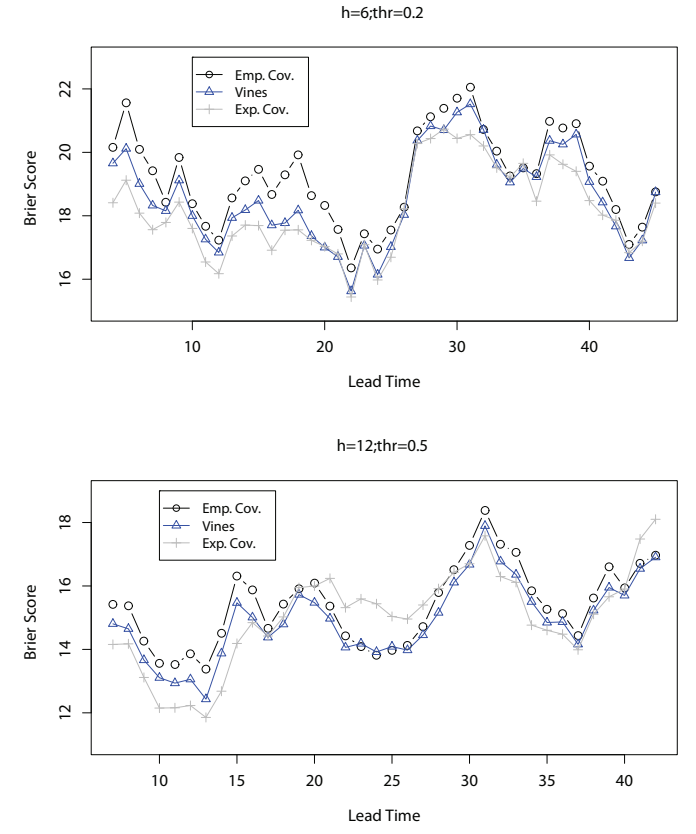

Figure 5. Brier score as a function of lead-time for wind farm 3.

\section{REFERENCES}

[1] R.J. Bessa, C.L. Moreira, B. Silva, M.A. Matos, "Handling renewable energy variability and uncertainty in power systems operation," Wiley Interdisciplinary Reviews: Energy and Environment, vol. 3(2), pp. 156178, March/April 2014.

[2] P. Haessig, B. Multon, H. Ahmed, S. Lascaud, P. Bondon, "Energy storage sizing for wind power: impact of the autocorrelation of dayahead forecast errors," Wind Energy, vol. 18(1), pp. 43-57, Jan. 2015.

[3] C. Monteiro, R.J. Bessa, V. Miranda, A. Botterud, J. Wang, and G. Conzelmann, "Wind power forecasting: state-of-the-art 2009," Report ANL/DIS-10e1, 2009.
[4] P. Pinson, H. Madsen, H. Aa. Nielsen, G. Papaefthymiou, B. Klöckl, "From probabilistic forecasts to statistical scenarios of short-term wind power production," Wind Energy, vol. 12(1), pp. 51-62, Jan. 2009.

[5] M.C. Cario, B.L. Nelson, "Modeling and generating random vectors with arbitrary marginal distributions and correlation matrix," Technical Report, Northwestern University, Evanston, IL, 1997.

[6] J.M. Morales, R. Mínguez, A.J. Conejo, "A methodology to generate statistically dependent wind speed scenarios," Applied Energy, vol. 87(3), pp. 843-855, March 2010.

[7] X. Ma, Y. Sun, H. Fang, "Scenario generation of wind power based on statistical uncertainty and variability," IEEE Transactions on Sustainable Energy, vol. 4(4), pp. 894-904, Oct. 2013.

[8] P. Pinson, R. Girard, "Evaluating the quality of scenarios of short-term wind power generation," Applied Energy, vol. 96, pp. 12-20, 2012.

[9] J. Tastu, P. Pinson, H. Madsen, "Space-time trajectories of wind power generation: Parametrized precision matrices under a Gaussian copula approach," Lecture Notes in Statistics: Modeling and Stochastic Learning for Forecasting in High Dimension, vol. 217, pp 267-296, 2015.

[10] P. Heejung, R. Baldick, D. Morton, "A stochastic transmission planning model with dependent load and wind forecasts," in IEEE Transactions on Power Systems, vol. 30(6), pp.3003-3011, Nov. 2015.

[11] S. Hagspiela, A. Papaemannouil, M. Schmid, G. Andersson, "Copulabased modeling of stochastic wind power in Europe and implications for the Swiss power grid," Applied Energy, vol. 96, pp. 33-44, 2012.

[12] G. Díaz, "A note on the multivariate Archimedean dependence structure in small wind generation sites," Wind Energy, vol. 17(8), pp. 1287-1295, Aug. 2014.

[13] H. Louie, "Evaluation of bivariate Archimedean and elliptical copulas to model wind power dependency structures," Wind Energy, vol. 17(2), pp. 225-240, Feb. 2014.

[14] D. Kurowicka, R.M. Cooke, Uncertainty Analysis with High Dimensional Dependence Modelling, New York: Wiley, 2006.

[15] E.C. Brechmann, C. Czado, S. Paterlini, "Flexible dependence modeling of operational risk losses and its impact on total capital requirements," Journal of Banking \& Finance, vol. 40, pp. 271-285, March 2014.

[16] H. Haghi, S. Lotfifard, "Spatiotemporal modeling of wind generation for optimal energy storage sizing," IEEE Transactions on Sustainable Energy, vol.6(1), pp.113-121, Jan. 2015.

[17] M. Scheuerer, T.M. Hamill, "Variogram-based proper scoring rules for probabilistic forecasts of multivariate quantities," Monthly Weather Review, vol. 143(4), pp. 1321-1334, April 2015.

[18] A. Sklar, "Fonctions de répartition à n dimensions et leurs marges," Publications de l'Institut de Statistique de L'Université de Paris, vol. 8, pp. 229-231, 1959.

[19] T. Bedford, R.M. Cooke, "Probability density decomposition for conditionally dependent random variables modeled by vines," Annals of Math. and Art. Intel., vol. 32(1-4), pp. 245-268, 2001.

[20] K. Aas, C. Czado, A. Frigessi, H. Bakken, "Pair-copula constructions of multiple dependence," Insurance: Mathematics and Economics, vol. 44(2), pp. 182-198, 2009.

[21] E.C. Brechmann, U. Schepsmeier, "Modeling dependence with C- and D-vine copulas: The R package CDVine," Journal of Statistical Software, vol. 52(3), pp. 1-27, 2013.

[22] R.J. Bessa, V. Miranda, A. Botterud, Z. Zhou, J. Wang, "Time-adaptive quantile-copula for wind power probabilistic forecasting," Renewable Energy, vol. 40(1), pp. 29-39, April 2012.

[23] V. Miranda, H. Keko, A. Duque, "EPSO: evolutionary particle swarms," Advances in Evolutionary Computing for System Design Studies in Computational Intelligence, vol. 66, pp. 139-167, 2007.

[24] Revolution Analytics and Steve Weston. doParallel: Foreach parallel adaptor for the parallel package. R package version 1.0.8 2014 .

[25] T. Hong, P. Pinson, S. Fan, "Global energy forecasting competition 2012," Inter. Journal of Forecasting, vol. 30(2), pp. 357-363, 2014.

[26] T. Gneiting, L. Stanberry, E. Grimit, L. Held, N. Johnson, "Assessing probabilistic forecasts of multivariate quantities, with an application to ensemble predictions of surface winds," TEST, vol. 17(2), pp. 211-235, 2008.

[27] P. Pinson, J. Tastu, "Discrimination ability of the energy score," Technical Report no. 15, Technical University of Denmark, 2013. 